\title{
Les cancers urogénitaux en région périphérique de Sénégal: à propos de 156 cas
}

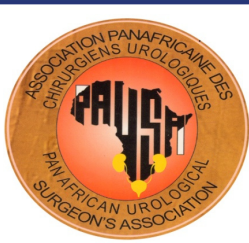

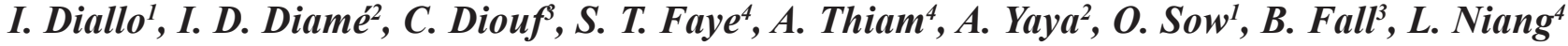 \\ ${ }^{1}$ Service de chirurgie générale hôpital, régional de Ziguinchor, ${ }^{2}$ Service d'Urologie-Andrologie hôpital régional d'Ourossogui, 'Département de chirurgie et spécialités chirurgicales UFRSS Université Assane Seck de Ziguinchor, ${ }^{4}$ Département de chirurgie et spécialités chirurgicales/Université Cheikh Anta Diop Dakar.}

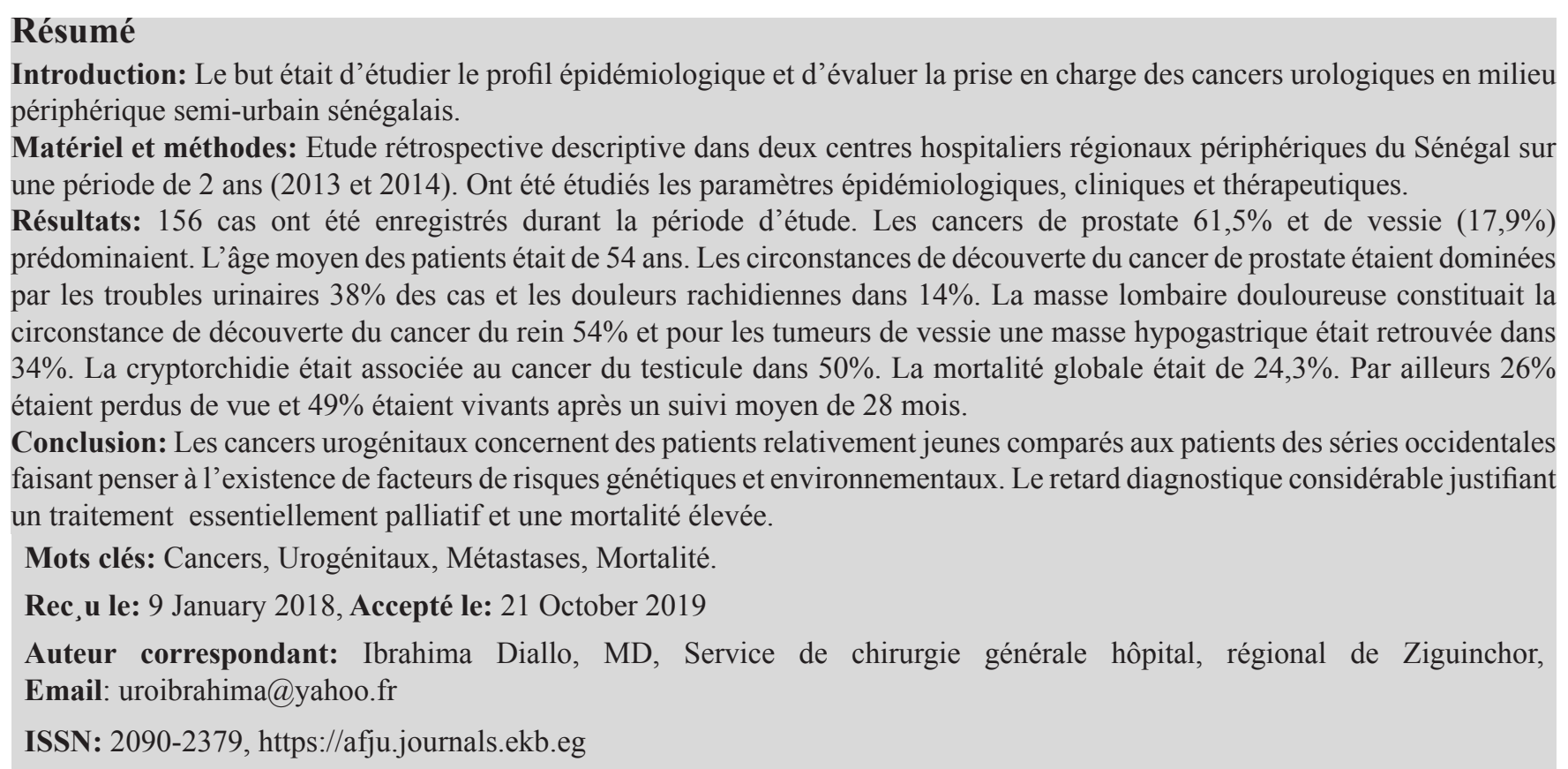

\section{Introduction}

Les cancers urogénitaux sont assez fréquents en Afrique $^{[1]}$. Cependant les données sur leur ampleur (incidence et prévalence), leur mortalité sont peu disponibles du fait de l'absence de registre de cancer dans la plupart des pays africains y compris au Sénégal. L'essentiel des études consacrées à ces cancers au Sénégal proviennent des structures hospitalières de référence du pays. Aucune étude à ce jour ne permet d'évaluer l'ampleur et la prise en charge de ces cancers au Sénégal. Ainsi ce travail se veut comme précurseur avec comme but d'étudier le profil épidémiologique et d'évaluer la prise en charge des cancers urologiques en milieu périphérique semi-urbain sénégalais.

\section{Matériels et méthodes}

Il s'agissait d'une étude rétrospective descriptive multicentrique menée dans deux centres hospitaliers régionaux périphériques du pays (Ziguinchor au sud du Sénégal et Ourossogui au Nord-Est). Nous avons inclus tous les patients ayant eu un cancer urogénital confirmé et hospitalisés dans les services urologiques de ces deux hôpitaux sur une période de 2 ans allant du 01 Février 2013 au 31 Décembre 2014.

Les paramètres suivants ont été étudiés:

- aspects épidémiologiques: type de cancer, âge, sexe

- aspects diagnostiques

- aspects thérapeutiques 


\section{Résultats}

\section{Aspects épidémiologiques}

Au total 156 cas de cancer urologiques ont été enregistrés durant la période d'étude. Les cancers les plus fréquents étaient celui de la prostate avec 96 cas $(61,5 \%)$ et celui de la vessie avec 28 cas (17,9\%). Il y avait 140 hommes et 16 femmes, soit un sex-ratio de 8,75 (Tableau I). L'âge moyen des patients était de 54 ans avec des extrêmes de 16 et 92 ans. La tranche d'âge la plus représentée était celle de 7079- ans (figure I). Un patient sur trois avait moins de 60 ans.

Concernant le cancer du rein, les femmes atteintes étaient plus jeunes (âge moyen 46,5ans ; extrêmes : 26 et 67 ans) que les hommes (âge moyen 55 ans; extrêmes 30 et 80 ans).
Pour le cancer de vessie, l'âge moyen des hommes était de 54 ans (extrêmes: 25 et 85 ans) et l'âge moyen des femmes était de 60 ans (extrêmes 30 et 85 ans).

Les patients atteints de cancer de prostate avaient un âge moyen de 69,9ans avec des extrêmes de 48 ans et 92 ans. La tranche d'âge de 7079- a été la plus touchée par le cancer de la prostate avec 58 cas soit $42.1 \%$. Il a été diagnostiqué deux fois plus de cancers de prostate chez les sujets de moins de 60 ans en 2014 qu'en 2013.

Le cancer du testicule était noté chez 6 patients. L'âge moyen était de 38 ans avec des extrêmes de 16 et 62 ans.

Les patients admis pour cancer de la verge étaient au nombre de 5. L'âge moyen était de 53 ans, avec des extrêmes de 27 ans et de 75 ans.

Tableau I: Répartition des patients selon l'organe atteint et le sexe

\begin{tabular}{lccccc}
\hline Années & & 2013 & & & 2014 \\
Sexe & Féminin & Masculin & Féminin & Masculin & TOTAL \\
\hline Prostate & - & 34 & - & 62 & 96 \\
Vessie & 4 & 8 & 4 & 12 & 28 \\
Rein & 3 & 7 & 5 & 6 & 21 \\
Verge & - & 1 & - & 5 & 6 \\
Testicule & - & 1 & - & 89 & 156 \\
TOTAL & 7 & 51 & 9 & & 5 \\
\hline
\end{tabular}

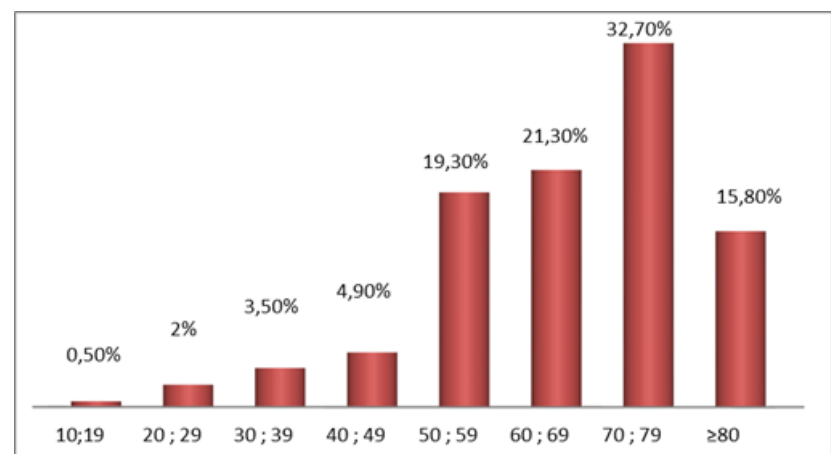

Fig. 1: Répartition des patients selon l'âge

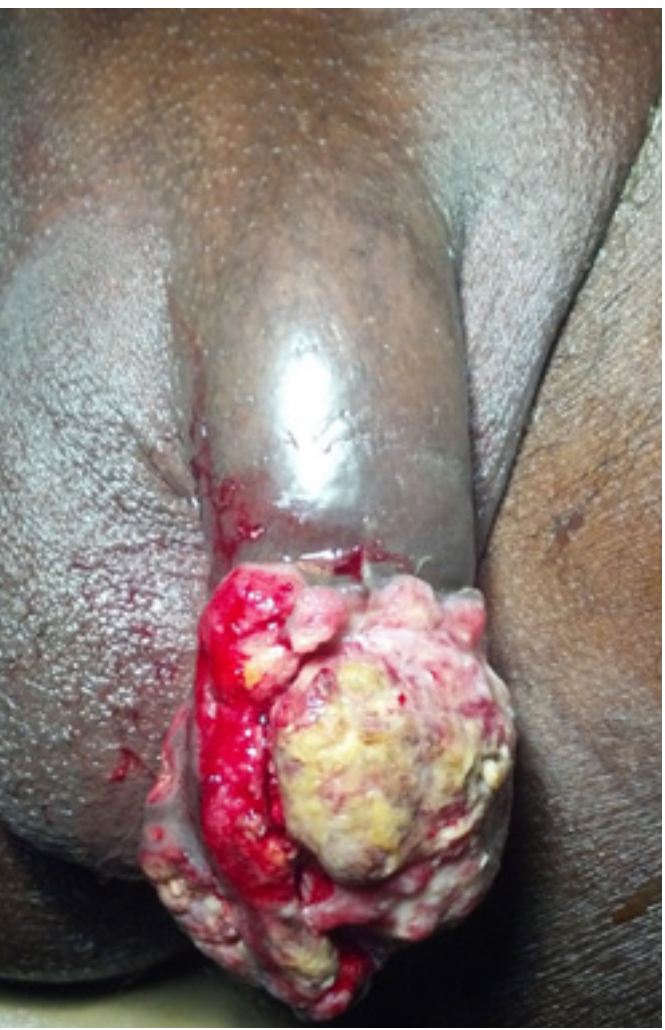

Cancer de verge T3 


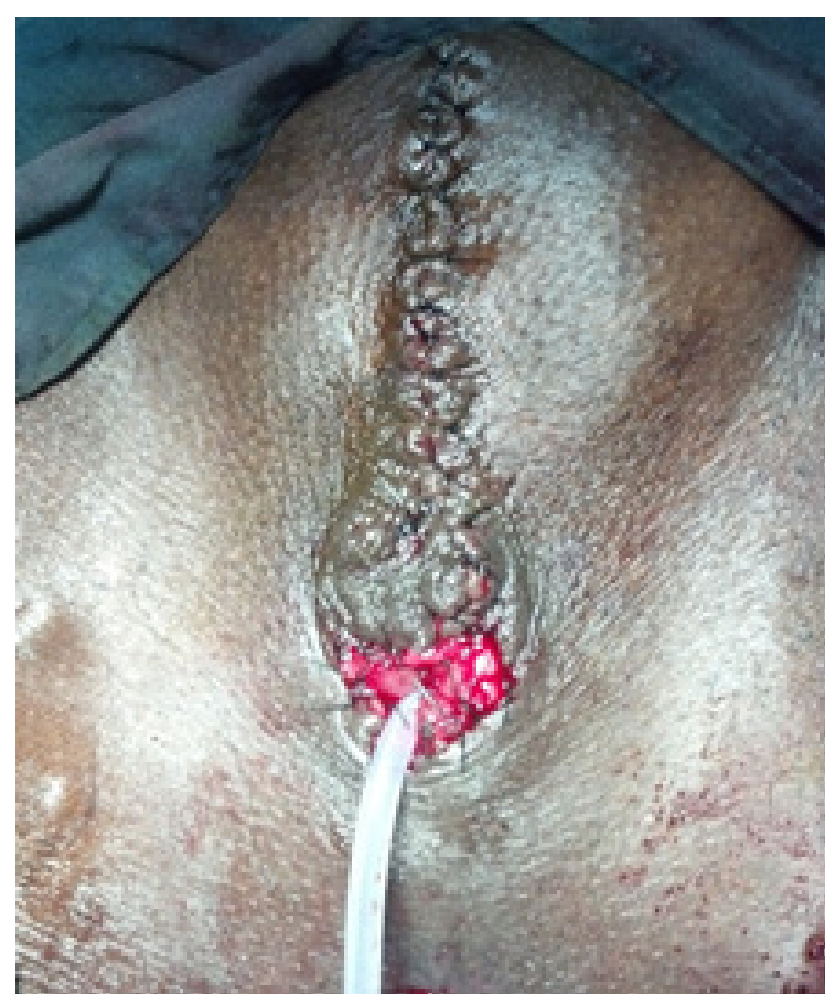

Aspect post-opératoire

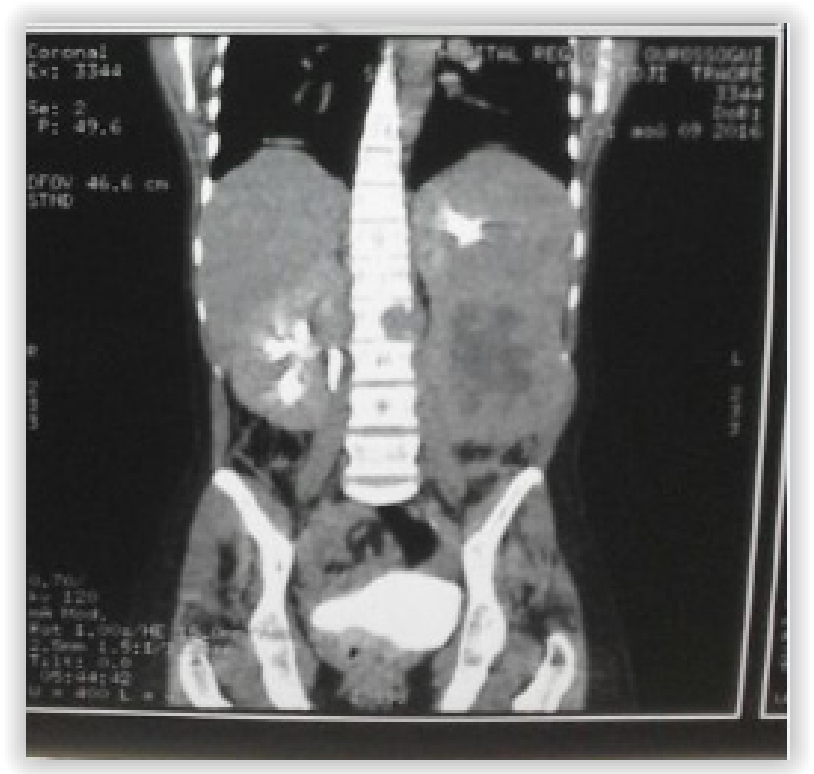

Tumeur rénale gauche $\mathrm{T} 4$

\section{Aspects diagnostiques}

$\square \quad$ cancer de la prostate

Les circonstances de découverte des cancers prostatiques étaient dominées par les troubles urinaires du bas appareil notamment une rétention complète d'urine dans $38 \%$ des cas. Les douleurs rachidiennes étaient révélatrices dans $14 \%$ des cas.
Le taux de PSA total moyen était de $1058 \mathrm{ng} / \mathrm{ml}$ avec des extrêmes de 6,2 et $19500 \mathrm{ng} / \mathrm{ml}$.

Le volume prostatique moyen était de 51,2 cc (28 et $160 \mathrm{cc})$.

La biopsie prostatique était faite chez 63 patients $(66 \%)$. Elle a permis de confirmer le diagnostic d'adénocarcinome qui était le type histologique dans tous les cas. Le cancer était bien différencié dans $43 \%$ des cas. Quatre-vingtquatorze patients $(60 \%)$ avaient des métastases au moment du diagnostic. Chez 22 patients (14\%), le cancer était localisé. Par ailleurs 40 patients $(25,6 \%)$ n'avaient pas d'investigations radiologiques nécessaires permettant de classer la tumeur.

\section{cancer du rein}

La douleur lombaire associée à une masse lombaire constituait la circonstance de découverte chez 13 patients (54\%). L'hématurie était présente chez 7 patients (29\%). L'URO-TDM et la tomodensitométrie thoraco-abdominopelvienne étaient les principaux examens d'imagerie réalisés chez nos patients. Ils ont permis de confirmer l'existence de la tumeur et de faire le bilan d'extension de celle-ci. La taille des tumeurs variait entre 6 et $25 \mathrm{~cm}$ de diamètre.

\section{$\square \quad$ cancer de la vessie}

L'hématurie était le principal motif de consultation (63\% des cas). La palpation d'une masse hypogastrique a été notée dans $34 \%$ des cas.

Le diagnostic a été posé par la TDM abdominopelvienne. Aucun de nos patients n'a eu une cystoscopie du fait de l'indisponibilité de l'endoscopie dans les deux centres.

\section{cancer du testicule}

Trois patients avaient une altération de l'état général associée à une grosse bourse non douloureuse. Trois patients avaient un cancer sur testicule non descendu intraabdominal. Chez ces patients, les signes cliniques étaient une masse abdominale associée à une vacuité de la bourse homolatérale et à une altération de l'état général. La TDM abdomino-pelvienne avait confirmé le diagnostic. Les marqueurs tumoraux, indisponibles n'ont pas été dosés.

\section{$\square \quad$ cancer de la verge}

Les 5 patients avaient consulté pour des lésions ulcérobourgeonnantes de la verge. Le diagnostic a été confirmé par la biopsie de la lésion. 


\section{Aspects thérapeutiques}

cancer du rein

Quatorze patients $(66,6 \%)$ avaient eu une néphrectomie élargie. Chez les 7 patients ayant des tumeurs très avancées avec altération profonde de l'état général, le traitement était palliatif, symptomatique.

cancer de la vessie

Une cystectomie partielle a été faite chez quatre patients et une pelvectomie antérieure avec Bricker chez une patiente. Quinze patients avaient eu une néphrostomie bilatérale pour des tumeurs trigonales avec urétérohydronéphrose bilatérale et insuffisance rénale obstructive. Dix patients ont été référés vers d'autres structures pour une meilleure prise en charge.

cancer du testicule

Une orchidectomie par voie inguinale a été faite chez 3 patients. Celle-ci était associée à une exérèse d'une masse ganglionnaire tumorale inguinale chez deux patients. Une laparotomie a été réalisée chez 2 patients avec exérèse tumorale. Un patient était décédé avant exploration chirurgicale. Aucun patient n'avait eu une radiothérapie ou une chimiothérapie.

\section{cancer de la verge}

Deux (2) patients ont été traités par une pénectomie partielle, avec marge de résection saine. Une émasculation associée à une urétrostomie périnéale a été faite chez les 3 autres patients.

\section{cancer de la prostate}

La prostatectomie radicale rétropubienne a été faite chez un patient. Il s'agissait d'un patient âgé de 61 ans ayant un cancer classé $\mathrm{T} 2 \mathrm{a}$ avec un score de Gleason à $7(34+)$ et un PSA total de 6,19 $\mathrm{ng} \backslash \mathrm{ml}$.

La suppression androgénique par castration chirurgicale a été le moyen thérapeutique le plus utilisé. En effet 68,3\% des patients avaient eu une pulpectomie testiculaire.

Un évidement Cervicoprostatique (ECP) a été fait chez 67 patients ayant une rétention vésicale d'urine. Dans 43 cas, il était associé à une pulpectomie testiculaire.

Un traitement antalgique a été instauré d'emblée chez $55 \%$ des patients. Il s'agissait d'antalgiques pallier II. Vingt-deux patients ayant une compression médullaire lente due à des métastases osseuses vertébrales ont été référés en neurochirurgie pour une meilleure prise en charge.

\section{Mortalité hospitalière}

Le taux de mortalité spécifique était de 24,3\%. Par ailleurs 41 patients $(26 \%)$ étaient perdus de vue et 77 patients (49\%) étaient vivants après un suivi moyen de 28 mois.

\section{Discussion}

\section{Données épidémiologiques}

En 2011, l'étude menée au Bénin par Ouattara A, et al avait objectivé une fréquence globale des cancers urogénitaux de $17,38 \%^{[1]}$. Il s'agissait de cancer prostatique $(69 \%)$, de vessie $(28,5 \%)$, du rein $(8,5 \%)$ et les cancers des organes génitaux externes ${ }^{[1]}$.

Notre étude révèle une prédominance des cancers prostatiques $(61,5 \%)$, suivis du cancer de vessie $(18 \%)$, et du cancer du rein $(13,4 \%)$.

Concernant l'âge et le sexe, nos résultats concordent avec ceux de Ouattara qui a rapporté un âge moyen des patients de $62,89 \pm 15,51$ ans, et $81,6 \%$ des patients ayant un cancer urologique avaient un âge supérieur à 50 ans. Aucun cancer pédiatrique n'a été retrouvé conformément aux études de Ouattara, de Gueye et de Ammani ${ }^{[1,2,3]}$. En effet le patient le plus jeune avait seize ans et présentait une tumeur séminomateuse du testicule.

L'âge moyen des patients atteints de cancer de prostate était de 69.9 ans, relativement identique aux résultats de Gueye et al sur une série de 121 patients, dont la moyenne d'âge était de 69 ans (52-88ans) ${ }^{[2]}$. Au Maroc, Ammani et al notaient une moyenne d'âge de 71ans (53-90) dans une étude qui incluait 258 patients $^{[3]}$. En France, Soulié et $a l^{[4]}$ évaluant l'incidence du cancer de la prostate, avaient objectivé une moyenne d'âge de 71,6 ans (46-94). Toutes ces données traduisent que le cancer de la prostate reste une maladie du sujet âgé.

\section{Aspects diagnostiques}

La révélation du cancer de la vessie, dans notre série, était surtout clinique avec une masse hypogastrique chez $34 \%$ des patients. Les mêmes constats ont été rapportés dans plusieurs séries africaines:DIAGNE au Sénéga ${ }^{[5]}$, COOPPAN en Afrique du Sud ${ }^{[6]}$, WAIHENYA au Kenya ${ }^{[7]}$. Devant l'indisponibilité de l'endoscopie, la TDM a permis de poser le diagnostic; et chez un patient elle a mis en évidence une tumeur unique siégeant au niveau du dôme qui a fait l'objet d'une cystectomie partielle. Cependant la résection trans urétrale de vessie avec examen anatomo-pathologique est nécessaire pour la confirmation histologique et la précision du caractère infiltrant ou non de la tumeur ${ }^{[8]}$. Le type histologique chez 
nos patients était le carcinome épidermoïde conformément à l'étude réalisée au Sénégal par Diao et $a l^{[8]}$.

Pour ce qui est du cancer de la prostate, lorsque celui-ci est localisé, il est asymptomatique et la présence de signes fonctionnels traduit un stade localement avancé ou métastatique comme démontré par Cussenot et Teillac ${ }^{[9]}$. Dans notre série, les circonstances révélatrices du cancer de la prostate étaient dominées par les troubles urinaires avec $83.6 \%$ des patients. Ammani et al avaient objectivé des manifestations urinaires chez $96,9 \%$ des patients. Dans l'étude d'Amégbor et al au Togo, ces manifestations urinaires représentaient $92 \%$ des cas ${ }^{[3]},^{[10]}$. Les symptômes extra-urinaires dans notre série étaient dominés par les douleurs rachidiennes dans $14 \%$ des cas. Cette fréquence des manifestations osseuses s'explique par le fait que les métastases dans le cancer de la prostate sont principalement osseuses $^{[11]}$.

Le dosage du PSA était important dans la démarche diagnostique. Ce dosage était réalisé chez tous nos patients, avec un taux moyen de $1058 \mathrm{ng} / \mathrm{ml}$. Dans l'étude de Guèye ${ }^{[2]}$ sur les caractéristiques cliniques du cancer de la prostate, le taux de PSA était également supérieur à la normale chez la population sénégalaise $(\mathrm{n}=121)$ avec des taux allant de 6 à 578,9. Pour Diallo et al ${ }^{[12]}$, en Guinée, sur une série de 203 patients, le taux moyen de PSA était de $120 \mathrm{ng} / \mathrm{ml}$ $(0,1-6354 \mathrm{ng} / \mathrm{ml})$. Ces données traduisent le retard diagnostic du cancer de la prostate dans notre contexte. L'échographie prostatique était réalisée chez tous nos patients et avait mis en évidence le caractère hétérogène de la prostate chez $81 \%$ des patients. Cependant, chez Esen et al, seuls $37,6 \%$ avaient un aspect hétérogène ${ }^{[13]}$.

Concernant la biopsie prostatique, $66 \%$ de notre échantillon ont eu une confirmation histologique. Dans la série de Diallo et coll[ ${ }^{[12]}$, en Guinée, tous les patients ont eu une confirmation histologique tout comme chez Amégbor et $a l^{[10]}$.

Toutefois, malgré une confirmation histologique de tous les patients des séries de Diallo et d'Amégbor en Afrique-subsaharienne, il y a un déficit en structures et en spécialistes. En outre, il a été constaté que la plupart des services d'anatomie pathologique sont situés dans les grandes villes. De plus, le délai d'attente des biopsies prostatiques est en général très long (environ 1 mois) pour des patients au stade métastatique. L'adénocarcinome était le type histologique retrouvé dans notre série. Les mêmes constatations sont retrouvées dans la littérature ${ }^{[2,10,12]}$.

S'agissant du score de Gleason, $43 \%$ de nos patients avaient un score $\leq 6$. Dans la série de Ammani et al $76 \%$ des patients ayant bénéficiés d'une confirmation histologique avaient un score de Gleason $\leq 6$.
La tumeur primitive était localisée au niveau de la prostate ou localement avancée chez $18,3 \%$ des cas. Ammani et coll. rapportait $48,83 \%$ des cas de cancer de la prostate colligés en 6 ans qui, au moment du diagnostic étaient localisés au niveau de la prostate ou localement avancée.

Les localisations secondaires étaient dominées par les métastases osseuses, cependant on notait une absence de bilan exhaustif. Cette prédominance des métastases osseuses vertébrales était retrouvée dans la littérature ${ }^{[14,15]}$. Ces métastases ont été diagnostiquées essentiellement par la tomodensitométrie $38,6 \%$ des patients, les radiographies standards 61,4\%, l'IRM et la scintigraphie osseuse n'avaient pas été réalisées. Au vue de ces données, on constate une faiblesse des investigations chez nos patients, surtout dans le cadre du bilan d'extension alors qu'il existait de nombreux signes extra-prostatiques. La tomodensitométrie était le moyen plus utilisé pour rechercher un envahissement locorégionale. Plusieurs auteurs comme Cussenot et Teillac pensent que la tomodensitométrie est peu efficace dans le diagnostic de l'extension extra-capsulaire, ils suggèrent plutôt l'IRM ${ }^{[9]}$. Néanmoins, dans notre contexte la tomodensitométrie reste toujours d'appoint, car l'IRM reste encore inaccessible.

\section{Aspects thérapeutiques}

La néphrectomie élargie avait été réalisée chez 14 de nos patients soit $66,6 \%$. Celle-ci constitue le traitement de référence des cancers du rein. La néphrectomie élargie est indiquée aussi bien dans un but de traitement radical que dans le cadre d'une réduction du volume tumoral ${ }^{[16]}$. Les voies d'abord utilisées dans notre série permettent toutes de respecter le contrôle premier du pédicule rénal qui est un impératif en matière de chirurgie des cancers du rein ${ }^{[16]}$. Aucun patient n'avait bénéficié d'un traitement ciblé à base de sunitinib, inhibiteur des tyrosinekinases, multicible. L'utilisation des antiangiogéniques a révolutionné le traitement du cancer du rein métastasé. En effet un essai thérapeutique de phase III comparant l'IFN $\alpha$ et le Sunitinib a révélé une survie sans progression de 23 semaines et 6 fois plus de réponses partielles en faveur du Sunitinib ${ }^{[17,18]}$. L'espoir réside dans l'utilisation de ces antiangiogéniques pour le traitement adjuvant des tumeurs localement avancées et les formes métastasées. Cependant le cout est hors de portée de nos populations.

Concernant le cancer de testicule, La littérature recommande une chimiothérapie précoce (J1) post orchidectomi $^{[19]}$. Aucun traitement adjuvant n'a été effectué chez nos patients. La chimio et la radiothérapie n'étant pas disponibles dans notre contexte de travail.

La prostatectomie radicale est l'un des traitements de référence du cancer de la prostate localisé chez les patients dont l'espérance de vie, estimée par l'âge et les 
co-morbidités, est supérieure ou égale à 10 ans ${ }^{[20]}$. Dans notre série la prostatectomie radicale a été réalisée chez 1 seul patient. La castration reste le traitement de référence dans la prise en charge thérapeutique du cancer de la prostate métastasé ; divers protocoles sont proposés pour l'hormonothérapie prostatique ${ }^{[21]}$. Dans notre série, la castration chirurgicale (pulpectomie) reste le traitement le plus utilisé. L'utilisation la pulpectomie dans notre contexte s'explique par son moindre coût, contrairement aux autres méthodes (castration médicale) qui sont relativement coûteuses et posant souvent des problèmes d'observance thérapeutique. En effetcelle-ciavait permis une amélioration clinique notamment de la douleur chez la plupart de nos patients. Ainsi, pour Bayoumi et coll, ${ }^{[22]}$ la pulpectomie permet une meilleure suppression androgénique sur le long terme avec un coût plus faible et offre ainsi une meilleure qualité de vie. De même, l'étude de VACURG rapportée par Bayoumi ${ }^{[22]}$ a démontré que la castration chirurgicale diminue la douleur et améliore l'état général chez les patients au stade avancé par rapport au placebo. Le BAC était instauré chez 32 patients. Selon Salomon et coll. ${ }^{[20]}$, l'utilisation de BAC par rajout d'un anti-androgène à la pulpectomie ou aux agonistes de la LH-RH ne pourrait être indiqué qu'en cas d'échappement hormonal. Par ailleurs, au cours de notre étude, il était difficile de faire la différence entre la résistance à la castration et la rechute par mauvaise observance thérapeutique, car aucun patient n'a bénéficié d'un dosage de la testostéronémie. Ainsi, 5 patients ont été considérés en échappement hormonal sur la base de la clinique et de l'élévation du taux du PSA sans que les autres critères définissant la résistance ne soient présents. La prise en charge des patients au stade d'échappement hormonal constitue un véritable défi du fait du manque d'un arsenal thérapeutique, surtout dans notre contexte socio-économique.

\section{Autres traitements (palliatifs)}

Prise en charge de la douleur

Dans notre série, $22,1 \%$ des patients avaient bénéficiés d'un traitement antalgique d'emblée, il s'agissait d'antalgique palier II. Malgré la fréquence des syndromes douloureux, aucun patient n'avait bénéficié d'une radiothérapie à visée antalgique. Au Cameroun, Yomi et coll $^{[23]}$, dans une étude portant sur 167 patients présentant des métastases cancéreuses et/ou des compressions tumorales ont obtenus un effet antalgique global dans $88 \%$ des cas et fonctionnelle dans $69 \%$ des cas avec une excellente tolérance et des complications minimes et un coût relativement peu élevé.

\section{Mortalité hospitalière}

Dans notre série, le décès a été constaté chez 26,7\% des patients au cours de l'hospitalisation. Au Bénin A. Ouattara et coll. rapportait $22,15 \%$ de décès liés aux cancers urologiques.

\section{Conclusion}

Les cancers urogénitaux concernent des patients relativement jeunes comparés aux patients des séries occidentales ce qui fait penser à l'existence de facteurs de risques génétiques et environnementaux. Le retard diagnostique est considérable avec une quasi inaccessibilité aux explorations paracliniques permettant un diagnostic et une stadification précis du cancer. On note également une inaccessibilité à certains traitements clés de la maladie cancéreuse (la radiothérapie, la chimiothérapie, les traitements antiangiogéniques) d'où un traitement essentiellement palliatif et une mortalité élevée.

\section{Intérêts concurrents}

Les auteurs déclarent n'avoir aucun conflit d'intérêts en relation avec cet article.

\section{Références}

[1] Ouattara A. et al.

Épidémiologie des cancers urologiques au Centre national hospitalier universitaire Hubert Koutoukou Maga Cotonou, Bénin. Analyse d'une série hospitalière de 158 cas. Prog Urol 2012, 22, 5, 261-265.

\section{[2]. Gueye SM, Jalloh M, Labou I et al}

Profil clinique du cancer de la prostate au Sénégal. African J of Urol 2004; 10(3): 203-207.

\section{[3]. Ammani A, Janane A, Chafiki J et al.}

Profil épidémiologique du cancer de la prostate dans le service d'urologie de l'hôpital Mohammed V de rabat.J Maroc Urol 2007; 5:11-14.

\section{[4]. Soulié M, Villiers A, Grosclaude P et al.}

Le cancer de la prostate en France : Résultats de l'enquête CCAFU- FRANCIM Prog Urol $2001 ; 11$ :478-485.

[5]. Diagne B.A., Ba M., Gueye S.M., Wandaogo A., Touré A., Mensah A. Les particularités des cancers vésicaux en milieu sénégalais : analyse de 336 observations. Bull Soc Fr Canc Prive 1987;16:95-100.

[6]. Cooppan R.M., Bhoola K.D., Mayet F.D. Schistosomiasis and bladder cancer in natal. S Afr Med J. 1984; 66(22): 841-3.

[7]. Waihenya C.G., Mungai P.N. Pattern of transitional cell carcinoma of the urinary bladder as seeb at Kenyatta National Hospital, Nairobi. East Afr Med J. 2004; 81(3): 114-9. 
[8]. Diao B, Thiam A, B Fall et al. Les cancers de vessie au Sénégal: particularités épidémiologiques, cliniques et histologiques. Progrès en urologie (2008) 18, 445-448.

[9]. Cussenot O, Teillac P. Le cancer de la prostate. Path. Sciences: Paris, 1999; 127:616.

[10]. Amégbor $\mathrm{K}$, Yao Seddoh $\mathrm{T}$, Tengué $\mathrm{K}$ et al. Epidémiologie et histopronostic du cancer de la prostate au Togo : à propos de 202 cas diagnostiqués au laboratoire d'anatomie pathologique du CHU Tokoin de Lomé. Prog Urol 2009; 19:112-115.

[11]. Sobin LH, Gospodariwicz M, Wittekind C. TNM classification of malignant tumors. UICC International Union against Cancer. 7th Edition. Wiley-Blackwell, 2009:243-8.

[12]. Diallo AB, YouweDombeu N, Barry A M et al. Caractéristiques cliniques du cancer de la prostate en Guinée. Résultats sur la période 2000-2006. African J of Urol. 2007, 13(4) :280-287.

[13]. Esen G. Ultrasound of superficial lymph nodes. Eur J Radiol 2006; 58:345-59.

[14]. Coleman R.E. Metastatic bone disease: clinical features, pathophysiology and treatment strategies. Cancer Treat. Rev.2001; 27:167-176.

[15]. Saad F, Clarke N, Colombel M. Natural history and treatment of bone complications in prostate cancer. EurUrol 2006; 49; 429-440.
[16]. Coulange C, Rambeaud JJ. Cancer du rein de l'adulte Rapport-Congrès AFU 1997. Prog Urol1997; 5.

[17]. MRC trial. Interferon-alpha and survival in metastatic renal carcinoma: early results of a randomised controlled trial. Medical Research Council Renal Cancer Collaborators. Lancet 1999; 353 (9146): 14-17.

[18]. Pyrhonen S, Salminen E, Ruutu M, et al. Prospective randomized trial of interferon alfa- $2 \mathrm{a}$ plus vinblastine versus vinblastine alone in patients with advanced renal cell cancer. J ClinOncol 1999; 17(9): 2859-67.

[19]. Houlgatte A., Vedrine L. Tumeurs germinales non séminomateuses. EMC (Elsevier Masson SAS, Paris). Urologie, 18-650-A-20, 2007.

[20]. Salomon L,Azria D, Bastide C.etal.Recommandations en onco-urologie 2010 : Cancer de la prostate Progurol 2010 ; 20 suppl. 4, S217-S252.

[21]. Allen N E, Appleby P, Key T J et al. Endogenous Hormones, Prostate Cancer Collaborative Group, Roddam A.W. Endogenous sex hormones and prostate cancer: a collaborative analysis of 18 prospective studies.J Natl Cancer Inst 2008; 100 (3): 170-183.

[22]. Bayoumi A.M., Brown A.D., Garber A.M et al. Cost-effectiveness of androgen suppression therapies in advanced prostate cancer. J Natl Cancer Inst 2000; 92: 1731-1739.

[23]. Yomi. J, Angwofo. III, Fouda. A et al. La radiothérapie, médicament essentiel de la douleur cancéreuse dans les pays en développement. Med d'Afrique Noire 1993; 40(5). 


\title{
Urogenital cancers in the peripheral region of Senegal: About 156
}

\section{cases}

\begin{abstract}
Introduction: The purpose of this study was to study the epidemiological profile and to estimate the coverage (care) of the urologic cancers in Senegalese semi-urban peripheral environment.

Material and Methods: It was about a descriptive retrospective study in two peripheral regional hospitals of Senegal over a period of 2 years (on 2013 and 2014). The epidemiological, clinical and therapeutic parameters were studied.

Results: All in all 156 urologic cases of cancer were registered during the period of study. Prostate cancers $61,5 \%$ and of the bladder $(17,9 \%)$ were the most frequent. The average age of the patients was of 54 years and the male sex was widely dominant. The diagnosis was put at a metastatic stage. So the circumstances of discovery of the cancer of prostate were dominated by the urinary disorders of the low device in particular a complete retention of urine in $38 \%$ of the cases. The spinal pains were revealing in $14 \%$ of the cases. The pain lumbar vertebra associated with a mass lumbar vertebra established the circumstance of discovery of the renal cancer $54 \%$ and for the tumors of bladder a hypogastric mass was found in $34 \%$. The cryptorchidism was associated with the cancer of the testicle in $50 \%$. The rate of global mortality was $24,3 \%$. Besides $26 \%$ were lost sight and $49 \%$ were alive after an average follow-up of 28 months.

Conclusion: The urogenital cancers concern relatively young patients compared with the patients of the western series what reminds the existence of genetic and environmental risk factors. The considerable diagnostic delay justifying an essentially palliative treatment explains the high mortality.
\end{abstract}

Keywords: Cancer, Urogenital, Metastasis, Mortality. 